\title{
Value of accelerated multimodality therapy in stage IIIA and IIIB non-small cell lung cancer
}

\author{
Malcolm M. DeCamp, MD ${ }^{\mathrm{a}, \mathrm{b}}$ \\ Thomas W. Rice, $M D^{\mathrm{a}}$ \\ David J. Adelstein, MD ${ }^{\mathrm{c}}$ \\ Mark A. Chidel, MD ${ }^{d}$ \\ Lisa A. Rybicki, MS \\ Sudish C. Murthy, MD, PhD \\ Eugene $\mathrm{H}$. Blackstone, $\mathrm{MD}^{\mathrm{a}, \mathrm{b}, \mathrm{e}}$
}

From the Department of Thoracic and Cardiovascular Surgery, ${ }^{a}$ the Transplant Center, Division of Surgery, ${ }^{\mathrm{b}}$ and the Departments of Hematology and Medical Oncology, ${ }^{\mathrm{c}}$ Radiation Oncology, ${ }^{\mathrm{d}}$ and Biostatistics and Epidemiology, ${ }^{\text {e }}$ The Cleveland Clinic Foundation, Cleveland, Ohio.

Read at the Eighty-second Annual Meeting of The American Association for Thoracic Surgery, Washington, DC, May 5-8, 2002.

Received for publication May 9, 2002; revisions requested July 8, 2002; revisions received Dec 3, 2002; accepted for publication Jan 14, 2003

Address for reprints: Malcolm M. DeCamp, MD, The Cleveland Clinic Foundation, 9500 Euclid Avenue/Desk F25, Cleveland, OH 44195 (E-mail: decampm@ccf.org).

J Thorac Cardiovasc Surg 2003;126:17-27

Copyright $(\odot) 2003$ by The American Association for Thoracic Surgery

$0022-5223 / 2003 \$ 30.00+0$

doi:10.1016/S0022-5223(03)00206-X
Objectives: This study was undertaken to assess accelerated multimodality therapy in patients with IIIA and IIIB non-small cell lung cancer in terms of toxicity, feasibility, response, survival, and recurrence (value) and to identify predictors of pathologic response and improved survival.

Methods: Between October 1994 and September 2000, a total of 105 patients with stage pIIIA $(n=78)$ or pIIIB $(n=27)$ non-small cell lung cancer were enrolled in a study of accelerated multimodality therapy, consisting of hyperfractionated radiotherapy with concurrent chemotherapy (paclitaxel and cisplatin) followed by resection and postoperative chemoradiation. Multivariable correlates of pathologic response and survival were assessed.

Results: Toxic effects related to induction therapy necessitated hospitalization in $40 \%$ of patients $(n=42)$; treatment-related mortality was $9 \%(n=9)$. With respect to feasibility, $100 \%$ of patients completed induction therapy, $93 \%(\mathrm{n}=98)$ of cancers were operable, $79 \%(\mathrm{n}=83)$ of cancers were curatively resectable, and $77 \%(\mathrm{n}=81)$ of patients completed all therapy. Sterilization of mediastinal nodes was similar $(P=.6)$ for $\mathrm{pN} 2(35 \%)$ and $\mathrm{pN} 3(30 \%)$ disease. Median, 2-year, and 5-year survivals were 27 months, 53\%, and 32\%, respectively. Locoregional recurrence, distant recurrence, and both were seen in $6 \%(\mathrm{n}=6), 45 \%(\mathrm{n}=47)$, and $3 \%$ $(n=3)$ of patients, respectively. Pathologic response was not predictable. Nodal status predicted incrementally decreasing survival for patients with cancers downstaged to ypN0 or ypN1 $(\mathrm{n}=35)$ versus ypN2 $(\mathrm{n}=44)$ versus ypN3 $(\mathrm{n}=20 ; P<$ .001 ). In addition, advancing age, squamous histologic type, and higher pT predicted poorer survival.

Conclusions: Accelerated multimodality therapy is equally valuable in IIIA and IIIB non-small cell lung cancers. Despite unpredictable response to induction therapy, younger patients and those with nonsquamous histologic type, sterilization of mediastinal lymph nodes, and lower pT benefit most. A ypN2 stage reduces but does not preclude long-term survival.

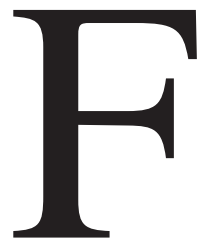
or the past decade, the approach to therapy for both stage IIIA and stage IIIB non-small cell lung cancer (NSCLC) at The Cleveland Clinic Foundation has differed from that of previous clinical trials. ${ }^{1-3}$ An accelerated multimodality protocol was implemented ${ }^{4,5}$ with the aims of improving feasibility, pathologic response, and survival and of reducing tumor recurrence with acceptable toxicity (value). Accelerated multimodality therapy consists of induction chemotherapy and concurrent hyperfractionated radiotherapy, ${ }^{6,7}$ resection with unilateral lymphadenectomy, and 
TABLE 1. Patient and tumor characteristics $(n=105)$

\begin{tabular}{lcr}
\hline Variable & No. & $\%$ \\
\hline Age (y) & & \\
Mean \pm SD & $57 \pm 10$ & \\
Median and range & $59(31-75)$ & 58 \\
Male (No.) & 61 & \\
Histologic type (No.) & & 38 \\
Adenocarcinoma & 40 & 37 \\
Large cell & 39 & 25 \\
Squamous & 26 & \\
Preinduction stage (No.) & & 17 \\
cT1 & 18 & 66 \\
cT2 & 69 & 70 \\
cT3 & 11 & 3 \\
cT4 & 7 & 77 \\
pN0 & 3 & 20 \\
pN2 & 81 & 74 \\
pN3 & 21 & 26 \\
IIIA & 78 & \\
IIIB & 27 & \\
\hline
\end{tabular}

accelerated adjuvant chemotherapy with concurrent hyperfractionated radiotherapy. Objectives of this study were to assess accelerated multimodality therapy in patients with stage IIIA and IIIB NSCLC in terms of toxicity, feasibility, tumor response, survival, and tumor recurrence (value) and to identify predictors of pathologic response and improved survival within this group.

\section{Patients And Methods \\ Patients}

Between October 1994 and September 2000, a total of 105 patients (58\% male, mean age $57 \pm 10$ years) with IIIA $(\mathrm{n}=78)$ or IIIB $(\mathrm{n}=27)$ NSCLC were enrolled in the study of accelerated multimodality therapy (Table 1). Only 7 (7\%) had cT4 disease (involvement of superior vena cava [ $\mathrm{n}=2]$ and mediastinum $[\mathrm{n}=5]$; surgical staging for these patients indicated $\mathrm{pN} 0$ disease in 3 cases, $\mathrm{pN} 2$ in 3 , and $\mathrm{N} 3$ in 1. Of the 105 patients, 102 (97\%) had stage III disease because of mediastinal nodal involvement.

\section{Eligibility and Staging}

All patients had histologic or cytologic diagnosis of NSCLC and were evaluated by a thoracic surgeon, medical oncologist, and radiation oncologist. Radiographic evaluation included chest radiographs; head, chest, and abdominal computed tomography; and radionuclide bone scanning. Beginning in 1999, whole-body positron-emission tomographic scanning with fluorodeoxyglucose tagged with fluorine 18 was used. Surgical staging was performed in all cases. Pretreatment stage was assigned according to American Joint Committee on Cancer lung cancer guidelines. ${ }^{8}$ Patients with stage III (pN2 or pN3) disease, as well as those with cT4 tumors, exclusive of malignant pleural effusions and separate tumor nodules in the same lobe, were included.

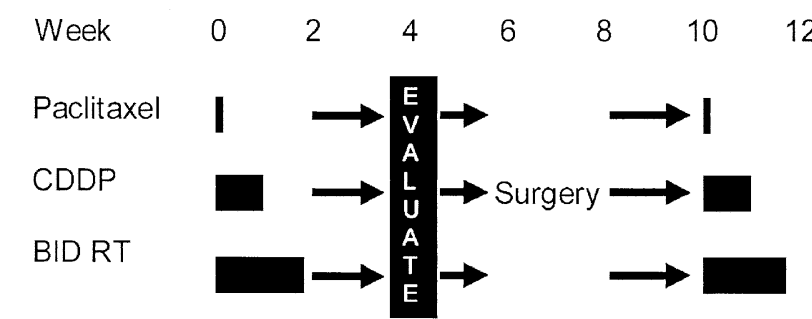

Figure 1. Treatment schema for accelerated hyperfractionated chemoradiotherapy followed by resection and adjuvant, consolidative chemoradiation for patients with stage IIIA and stage IIIB NSCLC. Cisplatin dose was $20 \mathrm{mg} \cdot \mathrm{m}^{-2} \cdot \mathrm{d}^{-1}$ for 4 days and paclitaxel dose was $175 \mathrm{mg} \cdot \mathrm{m}^{-2}$ as 24-hour infusion. CDDP, Cisplatin; BID RT, twice-daily radiotherapy at 1.5 Gy per fraction.

The study was approved by The Cleveland Clinic Foundation's Institutional Review Board and was reviewed annually. Written informed consent was obtained from each patient before therapy began.

\section{Treatment}

Accelerated induction therapy. Induction therapy consisted of a 12-day course of concurrent chemoradiotherapy, as previously reported elsewhere (Figure 1). ${ }^{5} \mathrm{~A}$ 4-day continuous infusion of cisplatin $\left(20 \mathrm{mg} \cdot \mathrm{m}^{-2} \cdot \mathrm{d}^{-1}\right)$ and a 24-hour infusion of paclitaxel $\left(175 \mathrm{mg} \cdot \mathrm{m}^{-2}\right)$ given on day 1 were administered to inpatients. Concurrent accelerated fractionation radiation therapy consisting of twice daily 1.5-Gy fractions given at least 6 hours apart were administered Monday through Friday during the first 2 weeks of treatment.

Four weeks after commencement of induction therapy, clinical reassessment included repeat chest computed tomographic scan and pulmonary function testing. Evidence of locoregional progression was defined as an increase greater than $25 \%$ in the sum of the crossed diameters of measurable tumor. Such progression or development of new distant metastatic disease excluded resection.

Resection. Resection consisted of lobectomy, bilobectomy, or pneumonectomy with complete unilateral mediastinal lymphadenectomy.

Accelerated adjuvant therapy. Within 6 to 8 weeks of resection, patients underwent a 2-week course of accelerated concurrent chemoradiotherapy similar to induction therapy (Figure 1). There were no chemotherapy dose reductions. Total adjuvant radiotherapy dose was between 30 and $33 \mathrm{~Gy}$. Total spinal cord dose was $<45 \mathrm{~Gy}$ with lateral and oblique fields; posterior spinal cord blocks were not used.

\section{Assessment}

Value of accelerated multimodality therapy was assessed in relation to its risks and benefits. 
Toxicity. Toxicity of induction and toxicity of adjuvant therapy were assessed by symptoms, signs, hospitalization, and related mortality. Surgical morbidity and mortality were included as components of toxicity.

Feasibility. Feasibility was assessed by ability of patients to complete induction therapy, operability, curative resectability, and ability to complete adjuvant therapy.

Tumor response. Tumor response was defined as downstaging of $\mathrm{cT}$ or $\mathrm{pN}$. Complete response was defined as ypTON0. ${ }^{8}$ Partial response was defined as reduction in cT or $\mathrm{pN}$ without a reciprocal increase in the other (progressive disease). For patients with $\mathrm{pN} 3$ disease, nonresponse was defined as ypN2.

Survival and tumor recurrence. Survival and tumor recurrence were assessed by patient follow-up. Patients were followed up every 3 months with clinical examination and chest radiographs. Additional testing was directed by clinical findings. Survivals from the time of commencement of induction therapy and after surgery were assessed by both the Kaplan-Meier method and a parametric method that resolves the number phases of instantaneous risk of death (hazard function). ${ }^{9}$ Recurrence was classified as locoregional (tumor within the ipsilateral thorax), distant (tumor outside the ipsilateral thorax), or both. The common closing date was October 17, 2001. Median follow-up was 18 months (range 2-79 months).

\section{Data Analysis}

Descriptive statistics included mean $\pm \mathrm{SD}$ and median and quartiles for continuous variables, with frequencies and percentages for categorical variables.

Predictors of pathologic response. Factors predictive of pathologic response were sought by multivariable logistic regression analysis with a stepwise selection procedure for which retention in the final model required $P<.05$. Factors considered potential predictors of pathologic response were age, gender, race, pretreatment $\mathrm{T}$, pretreatment $\mathrm{N}$, pretreatment stage, cell type, and clinical response.

Predictors of survival. The strategy to identify predictors of survival proceeded in two steps with multiphase hazard function methodology. ${ }^{9}$ At both steps we incorporated demographic characteristics, tumor histologic type, operative resection (pneumonectomy vs lobectomy), and date of therapy. Details are given in Appendix 1.

First, we focused on individual components of final pathologic TNM classification (ypTNM) and on the differences between pretreatment $\mathrm{cT}, \mathrm{pN}$, and ypTNM. We then added the variable pretreatment stage IIIB to test whether initial stage influenced survival beyond TNM classification. Second, we added to the previous the postresection stage grouping and pathologic response to treatment (complete, partial, none, or progressive disease). Then we again added the variable for pretreatment stage IIIB. The equation from
TABLE 2. Treatment-related toxicity

\begin{tabular}{lrr}
\hline Phase and type & No. & $\%$ \\
\hline Induction therapy (n= 105) & & \\
Nausea and vomiting & 92 & 88 \\
Grade III/IV & 4 & 4 \\
Mucositis or dysphagia & 88 & 84 \\
Grade III/IV & 11 & 10 \\
Neutropenia (<1000 cells $\cdot \mathrm{mm}^{-3}$ ) & 95 & 90 \\
Hospitalized & 42 & 40 \\
Renal & 5 & 5 \\
Toxicity-related death & 0 & 0 \\
Surgery (n = 98) & 7 & 7 \\
Mortality & 30 & 31 \\
Morbidity* & 18 & 18 \\
Supraventricular arrhythmia & 6 & 6 \\
Recurrent laryngeal nerve palsy & 3 & 3 \\
Pneumonia or adult respiratory distress syndrome & 3 & 3 \\
Bronchopleural fistula or prolonged air leak & 2 & 2 \\
Deep wound infection & 1 & 1 \\
Reoperation for bleeding & & \\
Adjuvant therapy (n = 84) $\dagger$ & 75 & 89 \\
Nausea and vomiting & 10 & 12 \\
Grade III/IV & 51 & 61 \\
Mucositis or dysphagia & 7 & 8 \\
Grade III/IV & 80 & 95 \\
Neutropenia (<1000 cells $\cdot$ mm ${ }^{-3}$ ) & 30 & 36 \\
Hospitalized & 1 & 1 \\
Myocardial infarction & 2 & 2 \\
Toxicity-related death & & \\
\hline
\end{tabular}

*Patients could have more than 1 complication.

tOf 98 patients undergoing resection, 84 received adjuvant therapy. An additional 4 patients with inoperable disease received adjuvant therapy.

this second multivariable analysis was solved for various combinations of risk factor values that illustrate the constellation of characteristics leading to good and to poor survival, as described by Kirklin and Barratt-Boyes. ${ }^{10}$

\section{Results}

\section{Value of Therapy}

Toxicity. During induction therapy, most patients experienced nausea, vomiting, mucositis or dysphagia, and neutropenia (Table 2), although grade III or IV symptoms were infrequent. Forty-two patients $(40 \%)$ required hospitalization for febrile neutropenia. There were no toxicity-related deaths from induction therapy. Although toxicity was common, it was manageable.

Surgical mortality was $7 \%$. Four patients died of pneumonia or adult respiratory distress syndrome, 1 died of postpneumonectomy bronchopleural fistula, 1 died of multisystem organ failure after perforation of a duodenal ulcer, and there was 1 sudden death at home within 30 days of surgery. Thirty patients (31\%) had postoperative complications (Table 2).

There were 2 toxicity-related deaths during adjuvant therapy, 1 from necrotizing pneumonia with subsequent 
TABLE 3. Pathologic response by preoperative stage

\begin{tabular}{crrrrr}
\hline \multirow{2}{*}{$\begin{array}{c}c \\
\text { Post-resection }\end{array}$} & \multicolumn{2}{c}{$\begin{array}{c}\text { Stage IIIA } \\
(\mathbf{n = 7 8 )}\end{array}$} & & \multicolumn{2}{c}{$\begin{array}{c}\text { Stage IIIB } \\
\text { (n= 27) }\end{array}$} \\
\cline { 2 - 3 } \cline { 5 - 6 } stage group & No. & $\%$ & & No. & $\%$ \\
\hline 0 & 10 & 13 & & 2 & 7 \\
I & 13 & 17 & & 5 & 19 \\
II & 4 & 5 & 1 & 4 \\
IIIA & 41 & 53 & 3 & 11 \\
IIIB & 5 & 6 & & 15 & 56 \\
IV & 5 & 6 & 1 & 4 \\
\hline
\end{tabular}

bronchopleural fistula and 1 from postemetic esophageal perforation. One patient had a nonfatal myocardial infarction related to occult coronary artery disease. Other toxic effects were similar to those of induction therapy (Table 2).

Feasibility. All patients completed induction therapy in 12 days (100\%). Ninety-eight patients (93\%) were operative candidates. Among the 7 inoperable cases, 4 patients had locoregional progression, 2 had new distant metastatic disease, and 1 was considered medically unfit for surgery.

Eighty-three patients (79\%) underwent curative resection. Of 15 patients who did not undergo curative resection, 6 had incomplete resection, 2 had residual N3 disease, 2 had intrapulmonary metastatic disease, and 5 had extensive involvement of the superior vena cava $(\mathrm{n}=3)$, descending aorta $(\mathrm{n}=1)$, or muscular chest wall $(\mathrm{n}=1)$. Curative resections included pneumonectomy $(\mathrm{n}=36)$, bilobectomy $(\mathrm{n}=5)$, and lobectomy $(\mathrm{n}=42)$.

Of the 91 operative survivors, 84 (92\%) commenced adjuvant therapy. Among the 7 who did not receive adjuvant therapy, 2 had protracted postoperative courses and 5 had disease progression. Among the 84 patients who began adjuvant therapy, 2 did not complete therapy because of toxicity, 1 died, and 1 had disease progression. One additional toxicity-related death occurred soon after adjuvant therapy was completed. Thus 81 of the 105 enrolled patients (77\%) completed and survived accelerated multimodality therapy.

Tumor response. There were no clinically determined complete responses, probably because of the brief interval between induction therapy and reassessment. Sixty-two percent of patients had a measurable partial response, defined as a more than $50 \%$ reduction in the sum of the crossed diameters of measurable tumor. Thirty-one percent had stable disease, and $6 \%$ had progression. Pathologic examination of the resected tissue demonstrated a complete response in 12 patients $(11 \%)$, a partial response in $43(41 \%)$, stable disease in $33(31 \%)$, and progressive disease in $17(16 \%)$.

Overall, 35 patients had sterilization of mediastinal lymph nodes (downstaged; Table 3 ). This response occurred in similar proportions of patients with IIIA $(\mathrm{n}=27 / 78$,
$35 \%)$ and IIIB $(\mathrm{n}=8 / 27,30 \%)$ disease $(P=.6)$. Postresection tumor classifications are listed in Appendix Table 1.

Survival. Median, 1-, 3-, and 5-year survivals for all patients from commencement of therapy were 27 months, $67 \%, 39 \%$, and $30 \%$. Survivals of the 98 patients undergoing operation were $70 \%, 42 \%$, and $26 \%$ at 1,3 , and 5 years (Figure 2, $A$ ). The instantaneous risk of death (hazard function) is depicted in Figure 2, B. It consisted of an initial period of high risk lasting approximately 6 months and a constant hazard of $25 \%$ per year thereafter. Median survival among the 15 patients who did not undergo curative resection was 18 months. Survivals of the 81 patients who completed multimodality therapy were $77 \%, 42 \%$, and $39 \%$ at 1,3 , and 5 years.

Tumor recurrence. Fifty-six patients had recurrent NSCLC, and 47 of them had died as of close of follow-up. Six recurrences $(6 \%)$ were locoregional, $47(45 \%)$ were distant metastatic, and $3(3 \%)$ were both.

\section{Predictors of Pathologic Response}

No pretherapy patient, staging, or tumor factor was predictive of pathologic response.

\section{Predictors of Survival}

The most important predictor of survival was pathologic stage $(P<.001$; Figure 3$)$. Patients with disease downstaged to ypN0 (pathologic stages $0, \mathrm{I}$ ) or ypN1 (pathologic stage II) had 55\% $\pm 10 \%$ 5-year survival, whereas patients with persistent stage IIIB disease were unlikely to survive 2 years $(18 \% \pm 9 \%)$. A statistically distinctive and novel intermediate cohort in this study was the group with residual stage IIIA, ypN2 disease. These patients had a median survival of 27 months, with $31 \% \pm 9 \%$ 5-year survival.

In the first analysis of ypTNM, incremental risk factors for death included older age, larger tumor after induction therapy (higher ypT), more extensive regional lymph node metastases (higher ypN), and squamous cell carcinoma (Table 4). After accounting for these factors, survival was unaffected by whether the disease had been stage IIIA or IIIB before treatment $(P=.8)$ or by the magnitude of reduction of $\mathrm{T}$ or $\mathrm{N}$ with induction therapy $(P=.15$ and $P=.8$, respectively).

The second analysis, including postresection stage grouping, added to the previously mentioned risk factors' lack of pathologic response to induction therapy and postresection stage IIIB and IV (ypIIIB/IV; Table 4). Survival benefit was equivalent for patients with partial and complete response. Pretreatment stage IIIA versus IIIB was not a predictor of survival $(P=.6)$.

A young patient with either large cell cancer or adenocarcinoma whose tumor was sterilized (downstaged) with induction therapy had excellent survival (Figure 4), regardless of whether the clinical stage was IIIA or IIIB before 

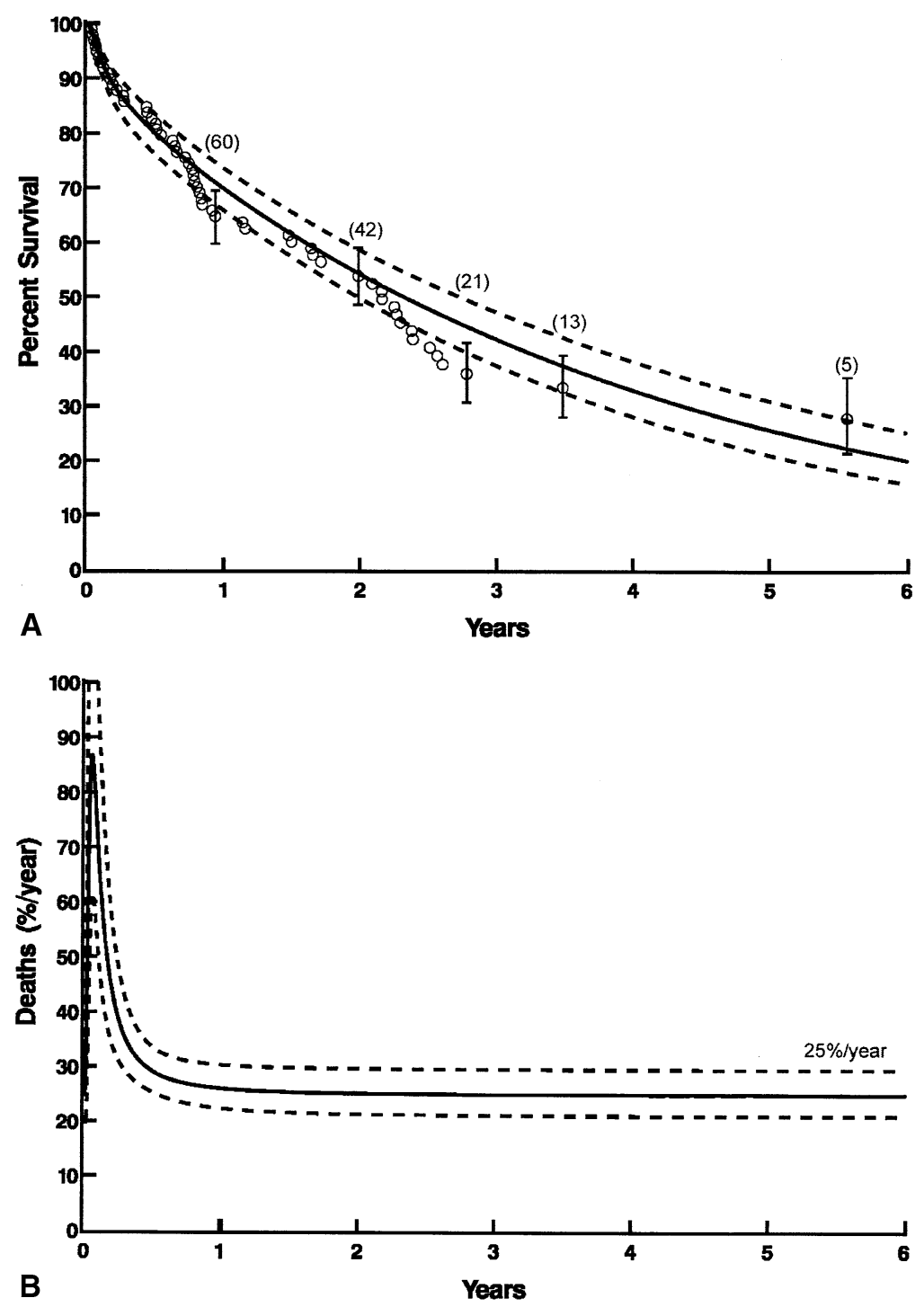

Figure 2. Postoperative survival after accelerated chemoradiotherapy. A, Survival. Each circle represents death positioned on vertical axis according to Kaplan-Meier estimator. These are accompanied by vertical bars representing asymmetric $68 \%$ confidence limits (equivalent to $\pm 1 \mathrm{SE}$ ). Numbers in parentheses represent survivors at that interval. Smooth curve is parametric estimate of survival enclosed within dashed asymmetric $68 \%$ confidence bands. B, Hazard. Instantaneous risk of death (hazard function) is represented by solid curve enclosed within dashed $68 \%$ confidence limits. Although early risk is high, this hazard is of short duration, resulting in few early deaths.

treatment. However, an older patient with squamous carcinoma that did not respond to treatment and continued in pathologic stage IIIB had limited survival (Figure 4).

\section{Discussion}

\section{Value of Therapy}

Value of therapy is an important but abstract concept that is difficult either to rigorously define or to quantify. However, it focuses attention on risks and benefits of therapy. Although a rigorous mathematical description and evaluation of value cannot be formulated, many of the medical components of value can be measured, even if not weighted, and predictors of value, such as favorable response, can be sought.

Toxicity (risk). Induction therapy followed by surgery was toxic, but toxicity was manageable and not fatal. Despite toxicity, surgery was not delayed, and surgical mortality was comparable to that with other protocols. Morbidity occurred in nearly every third patient, but more than half were atrial arrhythmias. Thus although morbidities were 


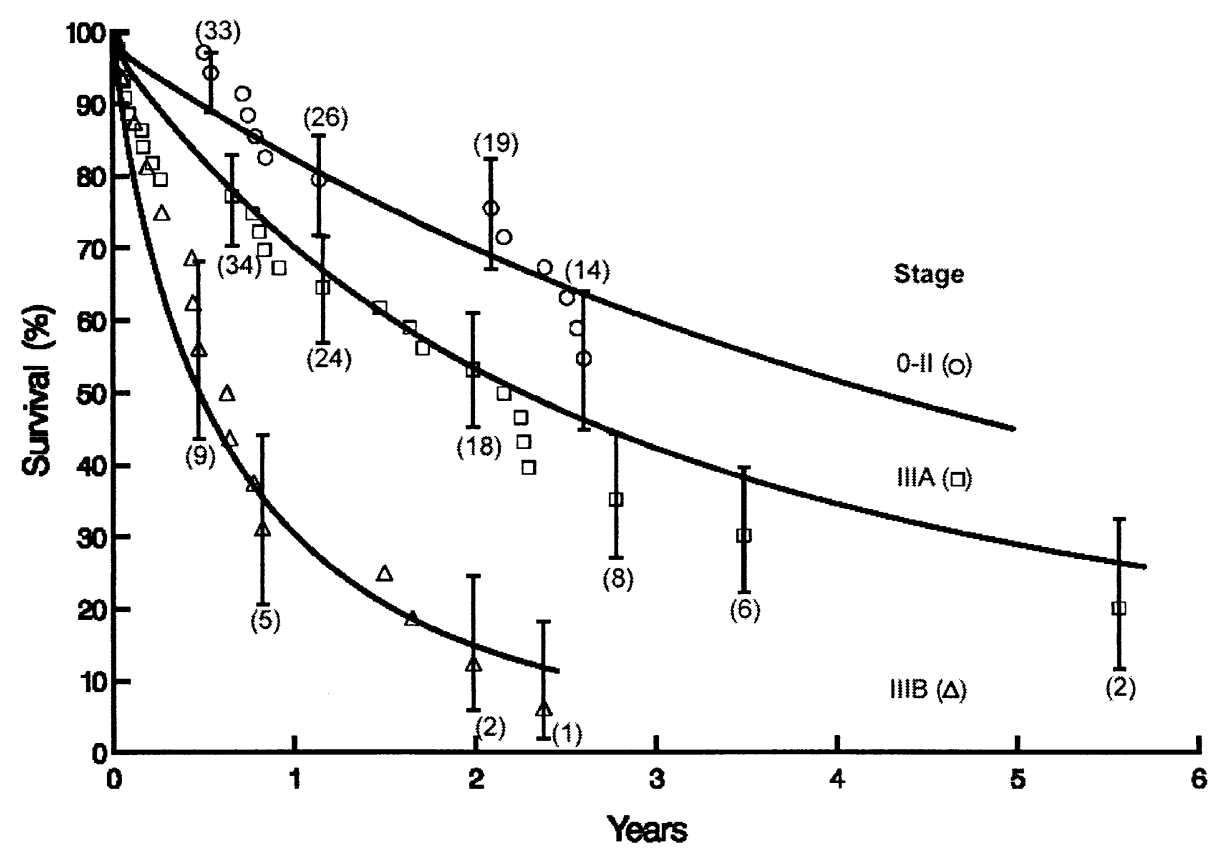

Figure 3. Survival by pathologic stage. Both Kaplan-Meier (symbols) and parametric (curves) survival estimates are depicted as in Figure 2, A. Parametric estimates are truncated when remaining survivors are few. Patients with pathologic stage 0 to II had survival superior to those with residual N2 disease (stage IIIA), who in turn had longer survival than did nonresponders (stage IIIB, $P<.001$ ).

\section{TABLE 4. Incremental risk factors for death after resection}

\begin{tabular}{|c|c|c|c|}
\hline Risk factor & Coefficient \pm SD & $\boldsymbol{P}$ & Reliability (\%) \\
\hline \multicolumn{4}{|l|}{ Analysis 1} \\
\hline Older age* & $-3.8 \pm 1.06$ & .0004 & 75 \\
\hline Larger tumor (урT) & $0.76 \pm 0.146$ & $<.0001$ & 70 \\
\hline More extensive regional lymph node metastasis (ypN)† & $0.022 \pm 0.0086$ & .01 & 68 \\
\hline Squamous cell carcinoma & $0.69 \pm 0.32$ & .03 & 53 \\
\hline \multicolumn{4}{|l|}{ Analysis 2} \\
\hline Older age* & $-3.4 \pm 1.09$ & .002 & 72 \\
\hline Larger tumor (ypT) & $0.33 \pm 0.195$ & .09 & 55 \\
\hline yplIIB/IV & $0.82 \pm 0.38$ & .03 & 62 \\
\hline Squamous cell carcinoma & $0.81 \pm 0.32$ & .01 & 78 \\
\hline No pathologic response to induction chemoradiotherapy§ & $-0.99 \pm 0.34$ & .003 & 56 \\
\hline
\end{tabular}

*Inverse transformation (50/age).

†Natural exponential transformation exp (ypN).

$\ddagger$ Postresection stage after induction therapy.

$\S$ Survival benefit.

multiple, they seemed not to be excessive. Toxicity of adjuvant therapy was similar to that of induction therapy, but in this final phase of therapy, toxicity-related deaths did occur. The overall observed treatment-related mortality of $9 \%$ compares favorably with the $10 \%$ reported by the multicenter Southwest Oncology Group (SWOG), which followed a more standard protocol of induction chemoradiation and resection. ${ }^{1}$

Feasibility. Induction therapy followed by resection was feasible. Resectability in $79 \%$ of cases is superior to rates reported after induction chemotherapy alone in stage IIIA N2 NSCLC ${ }^{11-13}$ and similar to the rate reported by SWOG after neoadjuvant chemoradiotherapy for both stage IIIA and stage IIIB disease. ${ }^{1}$ This accelerated strategy was feasible even though most cases of IIIB disease were $\mathrm{pN} 3$, unlike with SWOG 8805.

An advantage of accelerated multimodality therapy is superior patient compliance and less attrition as a result of accumulated toxic effects, increasing feasibility. Seventyseven percent of patients completed all prescribed treat- 


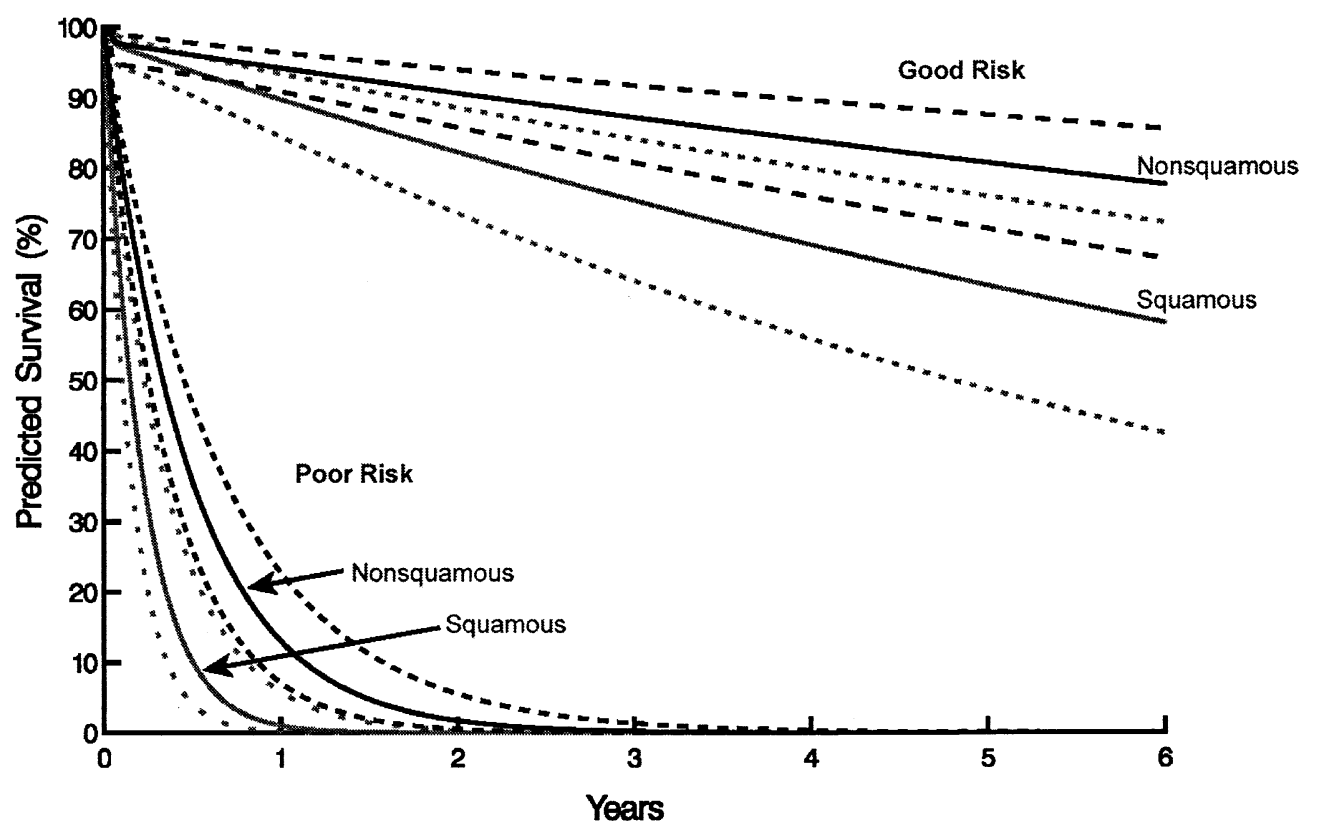

Figure 4. Good risk and poor risk patients. Shown at upper portion of graph is 45 -year-old patient whose adenocarcinoma was sterilized by accelerated chemoradiotherapy. For comparison, we present same patient profile except with squamous cell carcinoma. Survival at bottom of graph is for 65 -year-old patient who did not respond to therapy and was found to have T4 tumor at surgery. Two curves represent squamous and nonsquamous tumor histologic types.

ment, whereas only $45 \%$ of patients enrolled in Cancer and Leukemia Group B 8935 completed the prescribed therapy. ${ }^{11}$ Although a similar proportion of patients in SWOG 8805 (78\%) completed induction therapy, adjuvant therapy was prescribed and given to only $37(29 \%)$ of 126 trial participants. ${ }^{1}$

Response. Clinical response to accelerated induction therapy was similar to the response to three cycles of induction chemotherapy alone ${ }^{14}$ or to two cycles of concurrent chemoradiotherapy. ${ }^{1,15}$ The 12-day induction protocol had pathologic response (complete and partial) and nodal downstaging similar to those of other concurrent regimens lasting 33 days $^{15}$ and 36 days. $^{1}$

Survival. Accelerated multimodality therapy produced a median survival 1 year longer than that seen in SWOG 8805 , a phase II trial that also enrolled patients with both stage IIIA and IIIB disease. ${ }^{1}$ Long-term survival ( $>5$ years) was seen in $30 \%$ of patients, whereas it was $26 \%$ at 3 years in SWOG 8805. ${ }^{1}$

Recurrence. Local control was excellent, with only 9\% of patients having cancer recurrence in the ipsilateral thorax, compared with a $30 \%$ local recurrence rate reported by Grunenwald and colleagues ${ }^{15}$ in patients with IIIB disease and a $20 \%$ rate in SWOG $8805 .{ }^{1}$ Distant metastases remain the most common and discouraging problem. Nearly half of the patients had systemic recurrence, usually in the brain, a common finding with multimodality treatment of stage IIIA and IIIB disease. ${ }^{1,2,11-15}$

Predictors. Pathologic downstaging was the most important predictor of improved survival. Approximately a third of patients had their disease downstaged to N0 or N1, with a 5-year survival of almost 55\%. This is similar to survival among patients with newly diagnosed stage IB or stage IIA NSCLC. ${ }^{16}$ Mediastinal nodal sterilization provided equivalent survival regardless of whether the disease was initially N2 or N3.

Persistent mediastinal nodal metastasis has been reported as a reliable predictor of death, ${ }^{1,2,15}$ with $18 \% 3$-year survival. These results question the benefit of resection in the absence of nodal downstaging.

In contrast, this report establishes an important middle ground. Patients with residual N2 disease (ypN2 status) after accelerated induction therapy had a median survival of 27 months, with 31\% 5-year survival. One possible explanation for this near doubling of survival despite persistent $\mathrm{N} 2$ disease is the accelerated time frame. Indeed, some patients with microscopic residual nodal disease at the time of resection might in fact have had sterilization if several more weeks had elapsed before removal. This is reflected in a lower percentage of apparent pathologic downstaging in our study (33\%) than the $67 \%$ and $53 \%$ rates reported after standard induction therapy for $\mathrm{N} 2$ disease. ${ }^{1,2}$ However, the 
rate is similar to that observed after induction therapy for stage IIIB cancers. ${ }^{14,15}$

\section{Strengths and Limitations}

In this analysis we have highlighted the value of accelerated multimodality therapy for stage III NSCLC. It is difficult to know whether the enhanced overall survival is related to limited toxicity, increased feasibility, or better pathologic response. Hazard function analysis allowed treatmentrelated death to be distinguished from cancer-related death. ${ }^{9}$ In Figure 2, $B$, the area under the early, steep portion of the curve is the risk of toxicity-related death. Its height is governed by the toxicity of treatment (concurrent chemoradiation and surgery), and its width reflects duration of therapy. Accelerated therapy results in a brief period of treatment-related death risk. After completion of treatment, a steady-state risk of death reflects the ongoing and unremitting effects of residual or recurrent cancer.

Enhanced feasibility and response with manageable toxicity and prolonged survival are arguably the central strengths of accelerated multimodality therapy for both stage IIIA and stage IIIB NSCLC. These depend on dedicated multidisciplinary care and come with some unexpected costs, especially those related to unscheduled hospital readmission. The demonstrated feasibility of delivering all components of prescribed therapy is a testament to the expertise of our multimodality team and the increased stamina of selected patients who are treated rapidly. Experience and clinical volume translate into better outcomes in many realms of surgery. ${ }^{17}$ Requirements of experienced medical and radiation oncologists and general thoracic surgeons may limit the application of accelerated multimodality therapy to large-volume centers.

\section{Clinical Inferences}

Accelerated multimodality therapy is equally valuable in stage IIIA and stage IIIB disease, but pathologic response cannot be predicted. Patients with N2 and N3 disease respond similarly. Improved survival is seen in younger patients and those with nonsquamous histologic type, sterilization of mediastinal lymph nodes, and lower ypT. Persistent ypN2 disease reduces the probability of long-term survival but does not preclude it.

We thank Diane Baisden for database management, Barbara Chonko and Tess Knerik for editorial assistance, and Brian Kohlbacher for graphics expertise.

\section{References}

1. Albain KS, Rusch VW, Crowley JJ, Rice TW, Turrisi AT 3rd, Weick $\mathrm{JK}$, et al. Concurrent cisplatin/etoposide plus chest radiotherapy followed by surgery for stages IIIA (N2) and IIIB non-small-cell lung cancer: mature results of Southwest Oncology Group phase II study 8805. J Clin Oncol. 1995;13:1880-92.

2. Choi NC, Carey RW, Daly W, Mathisen D, Wain J, Wright C, et al.
Potential impact on survival of improved tumor downstaging and resection rate by preoperative twice-daily radiation and concurrent chemotherapy in stage IIIA non-small-cell lung cancer. J Clin Oncol. 1997; 15:712-22.

3. Andre F, Grunenwald D, Pignon JP, Dujon A, Pujol JL, Brichon PY, et al. Survival of patients with resected N2 non-small-cell lung cancer: evidence for a subclassification and implications. J Clin Oncol. 2000; 18:2981-9.

4. Rice TW, Adelstein DJ, Koka A, Tefft M, Kirby TJ, Van Kirk MA, et al. Accelerated induction therapy and resection for poor prognosis stage III non-small cell lung cancer. Ann Thorac Surg. 1995;60:58691.

5. Rice TW, Adelstein DJ, Ciezki JP, Becker ME, Rybicki LA, Farver $\mathrm{CF}$, et al. Short-course induction chemoradiotherapy with paclitaxel for stage III non-small-cell lung cancer. Ann Thorac Surg. 1998;66: 1909-14.

6. Thames HD Jr, Peters LJ, Withers HR, Fletcher GH. Accelerated fractionation vs hyperfractionation: rationales for several treatments per day. Int J Radiat Oncol Biol Phys. 1983;9:127-38.

7. Withers HR. Biologic basis for altered fractionation schemes. Cancer. 1985;55:2086-95.

8. American Joint Committee on Cancer. AJCC cancer staging manual. 6th ed. New York: Springer-Verlag; 2002.

9. Blackstone EH, Naftel DC, Turner ME Jr. The decomposition of time-varying hazard into phases, each incorporating a separate stream of concomitant information. J Am Stat Assoc. 1986;81:615-24.

10. Kirklin JW, Barratt-Boyes BG. Cardiac surgery. 2nd ed. New York: Churchill Livingstone; 1993. p. 249.

11. Sugarbaker DJ, Herndon J, Kohman LJ, Krasna MJ, Green MR. Results of cancer and leukemia group B protocol 8935. A multiinstitutional phase II trimodality trial for stage IIIA (N2) non-small-cell lung cancer. Cancer and Leukemia Group B Thoracic Surgery Group. J Thorac Cardiovasc Surg. 1995;109:473-83.

12. Roth JA, Fossella F, Komaki R, Ryan MB, Putnam JB Jr, Lee JS, et al. A randomized trial comparing perioperative chemotherapy and surgery with surgery alone in resectable stage IIIA non-small-cell lung cancer. J Natl Cancer Inst. 1994;86:673-80.

13. Rosell R, Gomez-Codina J, Camps C, Maestre J, Padille J, Canto A, et al. A randomized trial comparing preoperative chemotherapy plus surgery with surgery alone in patients with non-small-cell lung cancer. N Engl J Med. 1994;330:153-8.

14. Pitz CC, Maas KW, Van Swieten HA, de la Riviere AB, Hofman P, Schramel FM. Surgery as part of combined modality treatment in stage IIIB non-small cell lung cancer. Ann Thorac Surg. 2002;74:164-9.

15. Grunenwald DH, Andre F, Le Pechoux C, Girard P, Lamer C, Laplanche A, et al. Benefit of surgery after chemoradiotherapy in stage IIIB (T4 and/or N3) non-small cell lung cancer. J Thorac Cardiovasc Surg. 2001;122:796-802.

16. Mountain CF. Revisions in the international system for staging lung cancer. Chest. 1997;111:1710-7.

17. Birkmeyer JD, Siewers AE, Finlayson EV, Stukel TA, Lucas FL, Batista I, et al. Hospital volume and surgical mortality in the United States. N Engl J Med. 2002;346:1128-37.

18. Breiman L. Bagging predictors. Machine Learning. 1996;26:123-40.

\section{Appendix 1}

\section{Predictors of Survival}

Two aspects of identifying predictors of survival were unconventional, although, as has been pointed out to the readership of this Journal, they are rapidly becoming mainstream in statistical circles in one form or another: (1) careful consideration of the scaling of continuous and ordinal variables and (2) variable selection on the basis of random sampling (bootstrap bagging ${ }^{18}$ ).

For scaling, continuous and ordinal variables were assessed univariably by decile risk analysis to suggest a set of transformations of scale that may be required to ensure good calibration to 
Appendix TABLE 1. Pathologic response to accelerated chemoradiotherapy in IIIA and IIIB tumors

\begin{tabular}{|c|c|c|c|c|}
\hline \multirow{2}{*}{$\begin{array}{l}\text { Postresection tumor } \\
\text { classification }\end{array}$} & \multicolumn{2}{|c|}{$\begin{array}{c}\text { Stage IIIA } \\
(n=78)\end{array}$} & \multicolumn{2}{|c|}{$\begin{array}{c}\text { Stage IIIB } \\
(n=27)\end{array}$} \\
\hline & No. & $\%$ & No. & $\%$ \\
\hline \multicolumn{5}{|l|}{$\mathrm{ypT}^{*}$} \\
\hline 0 & 12 & 16 & 2 & 8 \\
\hline 1 & 28 & 37 & 9 & 35 \\
\hline 2 & 27 & 36 & 9 & 35 \\
\hline 3 & 2 & 3 & 3 & 12 \\
\hline 4 & 6 & 8 & 3 & 12 \\
\hline \multicolumn{5}{|l|}{ ypNt } \\
\hline 0 & 24 & 32 & 8 & 32 \\
\hline 1 & 5 & 7 & 1 & 4 \\
\hline 2 & 45 & 61 & 3 & 12 \\
\hline 3 & 0 & 0 & 13 & 52 \\
\hline
\end{tabular}

*Unknown in 4 cases.

tUnknown in 6 cases.

survival. Then 1000 bootstrap data sets were constructed and analyzed by stepwise forward selection with a criterion for retention of variables in the models of $P=.1$. The 1000 models were then subjected to analyses of correlated variables to quantify frequency of occurrence of each cluster of variables. The most frequently occurring member of a cluster appearing in at least $50 \%$ of the bootstrap analyses was selected for the final multivariable equation. The $50 \%$ rule equally balances false-positive and falsenegative identification of risk factors. For each continuous and ordinal variable selected by the $50 \%$ rule, the scaling that most frequently was observed in these 1000 models was selected as the transformation incorporated into the final model.

Tabular results of multivariable analyses include an expression of frequency of bootstrap occurrences (reliability of identification of risk factors). Regression coefficients rather than hazard ratios are presented because of the nonproportional nature of the multiphase hazard method and because of the transformations of scale required for continuous and ordinal variables.

\section{Discussion}

Dr Robert Ginsberg (Toronto, Ontario, Canada). I would like to congratulate Dr DeCamp and colleagues on a beautifully presented study. I have a few comments.

The title of the talk appears to assert that this induction chemoradiotherapy improves survival. I'm not sure that this has been proved, because there are no valid data for comparison. Be that as it may, I have a few questions for Dr DeCamp.

First, having had the experience of being in the SWOG study, was there any difference in selection of your patients with positive mediastinal lymph nodes? In other words, did you accept patients with single-station lymph node disease? If so, how many were there? Only 105 patients in a 6-year period were entered into this trial. What happened to the other patients with stage IIIA and IIIB disease at your institution during this time? How often was there multistation bulky nodal disease among your patients?

When you compare your data with those of SWOG to conclude that this accelerated chemoradiation improves survival, I have some questions. In your induction therapy all patients necessarily had to be hospitalized for their infusion, and $40 \%$ also had to be hospitalized because of toxicity. This occurred before the operation in the induction phase and after the operation in the adjuvant phase. Please comment on that and its comparison with the SWOG 4-week chemoradiation preoperative protocol. When you add up the figures, your protocol is a 12-week protocol, and many induction chemoradiation protocols nowadays are 4 to 5 weeks of induction chemoradiation followed by a 3- to 4-week rest, followed by surgery. That's a 10-week protocol.

Your resectability figures were unchanged, your patient compliance figures were unchanged, and your downstaging was unchanged relative to the SWOG trial—a very simple protocol, and the time of treatment, even though it was "accelerated hyperfractionation" of the radiotherapy, was identical to SWOG at 12 weeks.

When you say that you've improved survival, what's the certainty factor? It's a decade later, and certainly all of us have had patient selection changes in our induction therapy once we have experience with more patients. So how certain can you be that you've improved survival? Maybe you've just improved selection.

I also would like to know your center's philosophy on resection of T4 and N3 disease. At operation no attempt was made to dissect the N3 nodes. How do you know that you didn't have N3 disease left behind, even though the N2 nodes were sterilized?

Also, you had 7 T4 tumors, and none of these patients underwent an extended resection. Four of the T4 tumors were inoperable, and in the other 3 cases, as far as I can tell, there was no extended resection. All these patients underwent simple lobectomy or pneumonectomy. What is the philosophy of your surgical approach after induction chemoradiation? Does it not matter anymore that the original site of disease be removed? Is it okay just to remove the primary site of disease and the regional lymph nodes, leaving N3 nodes that may contain residual tumor?

Finally, now that you've prognosticated, how are you going to deal with the 60-year-old patient with squamous cell cancer? And how are you going to deal with the identification of persistent N2 disease, which you presume is also N3 disease, in the patients with initial N3 disease after the chemoradiation, since none of those patients survived?

Dr DeCamp. I appreciate your insightful comments, Dr Ginsberg, and certainly recognize your preeminence in this field.

Regarding your question as to our philosophy about singlestation versus multistation, intracapsular versus extracapsular, or bulky disease, we did not discriminate nor did we stratify our outcome analysis according to these factors. It is our philosophy that mediastinal nodal involvement is a marker for systemic disease, and without an effective systemic form of treatment as part of our treatment plan, we do not believe that we need to discriminate between those. All of that being said, I'm a firm believer that there are good IIIBs and bad IIIBs and good IIIAs and bad IIIAs; however, we tend to enroll all patients with reasonable performance status and any N2 or N3 involvement in this accelerated schema.

It is true that this is a toxic regimen. All of the patients are inpatients during the first week of both the induction and the adjuvant portions of treatment for continuous infusion chemotherapy and for adequate hydration to prevent renal toxicity. It's also 